\title{
Ultra-high frequency ultrasound (UHFUS) applications in Sjogren syndrome: narrative review and current concepts
}

\author{
Giacomo Aringhieri ${ }^{1}$, Rossana Izzetti ${ }^{2}$, Saverio Vitali ${ }^{3}$, Francesco Ferro ${ }^{4}$, Mario Gabriele ${ }^{2}$, \\ Chiara Baldini ${ }^{4}$, Davide Caramella ${ }^{1}$
}

${ }^{1}$ Diagnostic and Interventional Radiology, Department of Translational Research and of New Technologies in Medicine and Surgery, University of Pisa, Pisa, Italy; ${ }^{2}$ Unit of Dentistry and Oral Surgery, Department of Surgical, Medical and Molecular Pathology and Critical Care Medicine University of Pisa, Pisa, Italy; ${ }^{3}$ Diagnostic and Interventional Radiology, University Hospital of Pisa, Pisa, Italy; ${ }^{4}$ Department of Clinical and Experimental Medicine, University of Pisa, Pisa, Italy

Contributions: (I) Conception and design: All authors; (II) Administrative support: G Aringhieri, R Izzetti, S Vitali, F Ferro; (III) Provision of study materials or patients: G Aringhieri, R Izzetti, S Vitali, F Ferro; (IV) Collection and assembly of data: G Aringhieri, R Izzetti, S Vitali, F Ferro; (V) Data analysis and interpretation: All authors; (VI) Manuscript writing: All authors; (VII) Final approval of manuscript: All authors.

Correspondence to: Giacomo Aringhieri. Azienda Ospedaliero, Universitaria Pisana, Ospedale Cisanello, via paradisa, 2, 56124, Pisa, Italy.

Email: giacomo.aringhieri@unipi.it.

\begin{abstract}
Primary Sjogren's syndrome (SS) is a systemic autoimmune chronic inflammatory disease with predominant involvement of the exocrine glands, particularly the salivary glands (SGs). The role of salivary glands ultrasound (SGUS) in the work-up of patients with primary Sjogren syndrome (SS) is progressively increasing due to its useful support in diagnosis and follow-up as a widely available, repeatable, noninvasive and safe technique. Although SGUS is not yet included in the dominant primary SS classification, several studies supported its inclusion in the American College of Rheumatology/European League Against Rheumatism criteria. In this context, a novel imaging technique, ultra-high frequency ultrasound (UHFUS), is being explored. Compared to the frequencies used in conventional ultrasound (US) (up to $22 \mathrm{MHz}$ ), UHFUS operates with higher frequencies (30-100 MHz) allowing for outstanding image resolution, up to $30 \mu \mathrm{m}$. UHFUS permits the scan of both major and minor SGs, opening new avenues for the integration of tissue and imaging biomarkers. Although further studies are needed to confirm its role, this novel imaging technique might lead to several potential improvements, including earlier diagnosis, reduction of unnecessary and inadequate biopsies and better management and follow-up of patients with primary SS.
\end{abstract}

Keywords: Ultrasound (US); ultra-high frequency ultrasound (UHFUS); salivary glands (SGs); Sjogren syndrome (SS); imaging biomarkers

Submitted May 30, 2020. Accepted for publication Aug 27, 2020.

doi: 10.21037 /gs-20-529

View this article at: http://dx.doi.org/10.21037/gs-20-529

\section{Introduction}

Sjogren's syndrome (SS) is a systemic autoimmune disease characterized by the development of a functional impairment of exocrine salivary and lacrimal glands. The pathogenesis is related to the presence of a chronic lymphocytic infiltrate in the glandular parenchyma. Clinical presentation may be various, although the typical form of presentation includes xerostomia and xerophthalmia (sicca symptoms), which are present in the majority of patients (1). In younger patients, recurrent swelling of the parotid glands is also commonly reported (1). SS recognizes two forms of presentation. Primary SS (pSS) is characterized by the presence of sicca symptoms in absence of other systemic involvement, while secondary SS is characterized by the development of sicca symptoms in association with a concomitant systemic autoimmune disease $(1,2)$. Considering pSS, both sexes at all ages can be affected, 
although the disease is more often encountered in women between 30 and 50 years of age, with a prevalence range of $0.02-0.1 \%$ (2).

At the state of the art, the diagnosis of pSS is performed by a combination of clinical, serological, and histologic investigations, according to the American College of Rheumatology/European League Against Rheumatism (ACR/EULAR) criteria $(1,3)$. Clinical examination aims at identifying the presence of sicca symptoms. Laboratory tests support the assessment of autoimmune activation, with the presence of positive autoantibodies Ro/SSA and/or La/SSB. Finally, minor salivary gland (SG) biopsy provides histologic confirmation and evaluation of the severity of lymphocytic infiltrate in glandular parenchyma through focus score estimation.

Recent technological advances in head and neck imaging, including facial and oral imaging, and in particular SGs imaging, allowed to improve the multimodality study of this challenging anatomical region (4-12). The following step is to integrate patients' clinical and histopathology data with imaging biomarkers with the ultimate aim of using imaging biomarkers as decision-support tools to improve pSS diagnostic process and patients' stratification in clinical practice.

From this perspective, the role of salivary glands ultrasound (SGUS) has progressively increased, due to the several advantages of this easy-to-perform technique that is non-invasive, widely available and consistently repeatable. At the present, the dominant pSS classification systems do not include ultrasound (US) findings in their criteria (13), although their inclusion in the ACR/EULAR criteria has been suggested by different authors in place of sialography and salivary scintigraphy (14-17).

Noteworthy, in the very recent past, the use of ultra-high frequency ultrasound (UHFUS) has emerged in various clinical settings, including the study of skin, peripheral blood vessels and nerves, and oral mucosa, showing a significant correlation with the information deriving from tissue histology and opening new avenues for the integration of tissue and imaging biomarkers $(18,19)$.

Herewith, we provide a brief review of the literature about the role of imaging biomarkers in pSS focusing on conventional US of the major SGs and the technical background of UHFUS in order to discuss the current imaging concepts as well as the potential future applications of SGs UHFUS in the pSS clinical setting and research. We present the following article in accordance with the NARRATIVE REVIEW reporting checklist (available at http://dx.doi.org/10.21037/gs-20-529).

\section{Methods}

A literature search was performed in the PubMed record to write this narrative review. In particular, for conventional SGUS, only relevant review articles published from 1990 to 2020 were included. Given the limited number of publications regarding UHFUS, the search was extended to other databases (Web of Science and Google Scholar) to maximize the results and no relevant research work was excluded on the basis of year of publication, article type or study design. Only articles published in English language were included. Reference lists were also examined to find additional relevant articles.

\section{Discussion}

\section{SGUS in SS: a brief review}

Conventional SGUS is usually performed with linear-array probes with frequencies up to $22 \mathrm{MHz}(13,20-23)$. The main indications for SGUS as stated by American Institute of Ultrasound in Medicine (AIUM) include the presence of diffuse enlargement and tenderness consistent with inflammatory sialadenitis and recurrent swelling suggesting pSS are included (24). A recent review stated that SGUS is an accurate method to identify the pSS echostructural anomalies within the SGs with specificity and sensitivity of $0.91 \%$ and $0.83 \%$, respectively. Despite the clinical and methodological heterogeneity of the studies included in the review, these results are clinically interesting and highlight the significant contribution of SGUS in pSS diagnosis and follow-up (25-27). The variability of the interpretation of US images in patients with pSS still represents a limitation not yet overcome. However, imaging features like echogenicity and homogeneity were identified as reliable for pSS diagnosis, if assessed by a trained expert and evaluated in association with other classification criteria (28). While normal echostructure and homogeneity of SGs resembles the normal thyroid gland (hyperechoic compared to muscles), in affected glands echogenicity appears reduced by the presence of hypo/ anechoic areas and/or increased by fibrosis (linear-shaped) or fatty infiltration (focal/diffuse), with a progressive evolution from hypo- to hyperechoic alterations. Parenchymal inhomogeneity with heterogeneous echostructure typically occurs in all the major SGs in 
patients with $\mathrm{pSS}$, with demonstration of hypo/anechoic areas and hyperechoic foci/bands (28). Additionally, other US findings, such as cysts, calcifications, overall glands size variations, irregularities in glandular profiles and the presence of intra- or periglandular lymph nodes, may support pSS diagnosis (25).

The presence of bilateral changes in all the major SGs is the most relevant detectable US finding suggestive for pSS with the highest agreement with scintigraphy of the SGs, sialography, and minor labial salivary gland biopsy (LSGB). Finally, it has been suggested that in case of normal SGUS findings in patients with clinically suspected pSS with negative serum antibodies, can rule out pSS avoiding the LSGB (29). Indeed, SGUS findings have been evaluated as a predictor of the biopsy score. However, since the diagnosis of pSS is mainly based on the biopsy of minor SGs whereas conventional ultrasonography explores the echostructure of the major SGs the correlation between imaging biomarkers and minor SG histopathological grading remains moderate $(30,31)$.

Different SGUS-based semiquantitative scores have been proposed but their wide variability, including heterogeneous cut-offs and inhomogeneous parameters for image interpretation, led to inconsistent specificity and sensitivity $(20,25)$. However, the scoring system proposed by Jousse-Joulin et al. (21) seems to be effective in assessing SGs involvement with good diagnostic accuracy (25). Despite the promising results, further studies are needed to consolidate the role of SGUS as non-invasive tool for pSS diagnosis.

A role for prognosis estimation in pSS has also been claimed for SGUS due to the presence of a correlation between pathological US findings and increase in disease activity, risk of lymphoma, and development of systemic complications (32).

In addition to the SGUS morphologic evaluation, colour/power Doppler US (C/PDUS) can provide important information regarding glandular vascularization. Pathological C/PDUS findings in pSS include glandular hypervascularization, mainly with diffuse pattern: the small vessels within the glands are dynamically visible as punctiform areas of colour doppler signal. Indeed, hypervascularization showed a positive correlation with the parenchymal heterogeneity and the number of cyst-like alterations $(25,33)$. Interestingly, quantitative estimation of pulsatility and resistivity indexes showed a significant reduction in patients with the early phase of SS, presumably due to the vasodilatation caused by the active glandular inflammation (25). However, a global reduction of C/ PDUS signals was observed in patients with advanced pSS in association with an overall reduction of glandular volumes due to the limited active inflammation and to the increased fibrosis (25,33-35). Additionally, Martinoli et al. described the dynamic C/PDUS evaluation after lemon juice stimulation that caused increased vascular signal and decreased pulsatility and resistivity indexes in pSS pathological glandular parenchyma with subsequent normalization after about 20 seconds (33). Conversely, the facial artery in patients with pSS showed significantly lower changes in pulsatility and resistivity indexes compared to healthy controls (34). Among the C/PDUS parameters evaluated, Carotti et al. found that peak systolic velocity was more sensitive compared to the resistive index (36).

Recently, a new step towards the inclusion of SGUS in the 2016 ACR/EULAR pSS classification criteria has been made. Jousse-Joulin et al. (37) reported that SGUS had similar weight to other minor items already included in the 2016 ACR/EULAR pSS classification criteria, such as, namely, Schirmer test and unstimulated whole salivary flow rate. Moreover, including SGUS in the 2016 ACR/EULAR pSS classification criteria provided a higher sensitivity with no relevant change in specificity.

Nowadays, a consensus for a SGUS scoring system still represents an unmet need and the inclusion in the 2016 ACR/EULAR pSS classification criteria is not yet approved. However, SGUS examination with C/PDUS evaluation seems useful for the diagnosis and follow-up in patients with pSS and it is considered the first-time examination as widely available, repeatable, non-invasive, safe and low-cost investigation.

As in other clinical fields (18,38-43), UFHUS is being explored in the pSS scenario in order to evaluate its potential contribution to improve the evaluation of the SGs, particularly regarding minor SGs.

\section{UHFUS: technical notes}

UHFUS is a novel diagnostic technique which is finding an increasingly important role in several medical fields $(18,19,38-45)$, including ophthalmology (38), dermatology $(39,40)$, oral medicine $(18,19,44,45)$, musculoskeletal $(41,42)$ and vascular imaging $(43)$. Since the axial resolution achievable increases with the frequency, the use of frequencies between $30-100 \mathrm{MHz}$ allows to investigate superficial structures at a higher axial resolution compared to conventional US (46). The outstanding improvements in 

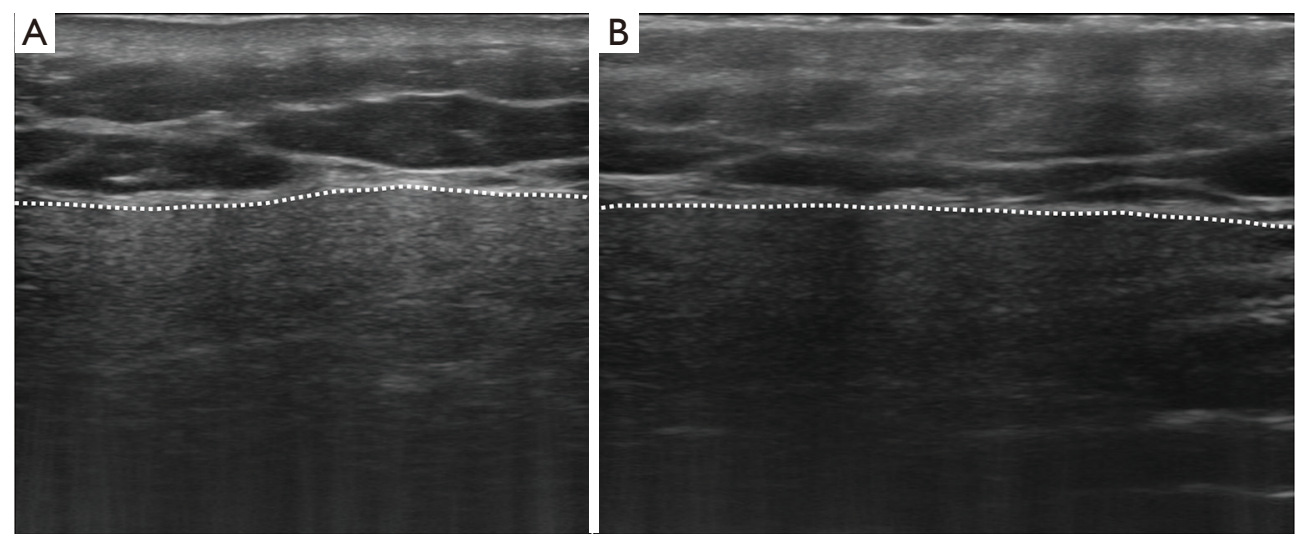

Figure 1 Normal parotid (A) and submandibular (B) glands depicted by using UHFUS with the $48 \mathrm{MHz}$ probe. Although the whole glandular structure cannot be imaged due to a limited depth of penetration of the ultrasound beam, high resolution provides a clear depiction of regular glandular margins (dotted line) and homogeneous parenchymal echostructure. UHFUS, ultra-high frequency ultrasound.

image quality are provided by the shorter US wavelength (inversely proportional to frequency). Nevertheless, the wavelength reduces the US penetration, limiting the thickness of the superficial tissues that can be evaluated with UHFUS (47). Thus, the most appropriate trade-off between resolution and penetration should be found for each different clinical application (48).

In our institution, Vevo MD (VisualSonics, Toronto, Canada) a UHFUS equipment is available since 2016; The UHFUS system is equipped with two probes: UHF48 operating up to $46 \mathrm{MHz}$ (center frequency $30 \mathrm{MHz}$ ), providing an image depth of $23.5 \mathrm{~mm}$ and an axial resolution of $50 \mu \mathrm{m}$, and UHF70 operating up to $71 \mathrm{MHz}$ (center frequency $50 \mathrm{MHz}$ ), with image depth of $10.0 \mathrm{~mm}$ and axial resolution of $30 \mu \mathrm{m}$. UHFUS applications in different clinical fields have been explored, including rheumatology.

The current concepts of UHFUS application in major and minor SGs evaluation are reported and discussed in order to analyse the potential contribution of this technique in patients with pSS. Further considerations based on our preliminary clinical experience are also reported and discussed.

\section{SGs UHFUS in SS: current concepts}

\section{Major SGs UFHUS}

UHFUS of the major SGs is performed with the $48 \mathrm{MHz}$ probe, similarly to conventional SGUS scan with both axial and longitudinal acquisitions of all the major SGs. Similarly to SGUS, normal
UHFUS appearance of salivary glandular tissues is characterized by homogeneous echogenicity with defined glandular margins (Figure 1). In pathologic glands, diffuse parenchymal inhomogeneity, hypoechoic areas, and hyperechoic foci/bands can be detected (Figures 2 and 3). In patients with suspected pSS, the typical alterations in glandular echostructure, in particular inhomogeneity, can be better depicted with UFHUS due to the increased axial resolution and improved contrastto-noise ratio (49). Thus, UHFUS of major SGs might improve diagnosis performance even at an early stage, allowing the identification of initial signs of inhomogeneity in the gland echostructure and of hypoechoic areas of small dimensions, which cannot be detected with conventional SGUS. Given the potential bimodal comparative evaluation that can be performed in major SGs, further comparison studies between SGUS and SGs UHFUS will permit to assess the role of SGs UHFUS in the reduction of false negative US examinations. It may be expected that the application of UHFUS to major SGs investigation may lead to an increase of the overall diagnostic accuracy in patient with pSS, particularly in the early phases. Indeed, the role of SGUS in the early diagnosis of pSS is still controversial (50). Although some authors stated that normal US findings in SGs have been observed in patients suffering from pSS for about 4.2 years (51), a recent study described the role of SGUS in the early diagnosis of pSS considering patients with symptoms duration inferior to 5 years (52). According to these considerations, the potential role of UHFUS in the early diagnosis of pSS should be further explored. 

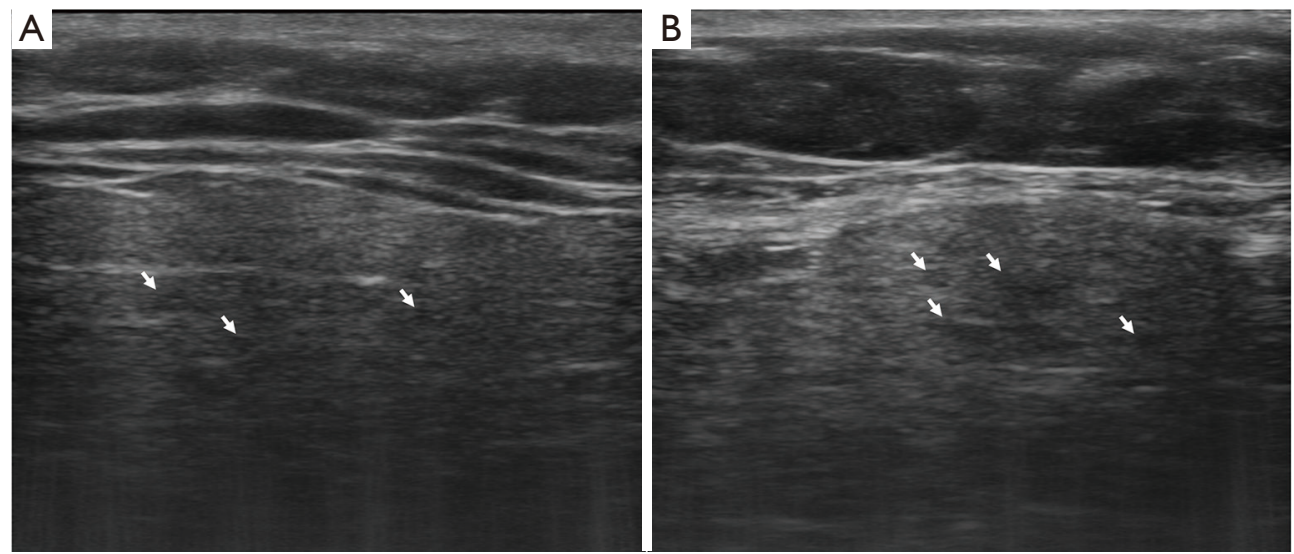

Figure 2 Mild alteration of the parenchymal echostructure of parotid (A) and submandibular (B) glands depicted by using UHFUS with the $48 \mathrm{MHz}$ probe. Unlike normal glands, initial phases of glandular inflammation secondary to pSS correspond to the presence of a fine inhomogeneity of the glandular parenchyma with occasional slightly hypoechoic areas with blurred edges (arrows). UHFUS, ultra-high frequency ultrasound; pSS, primary Sjogren's syndrome.
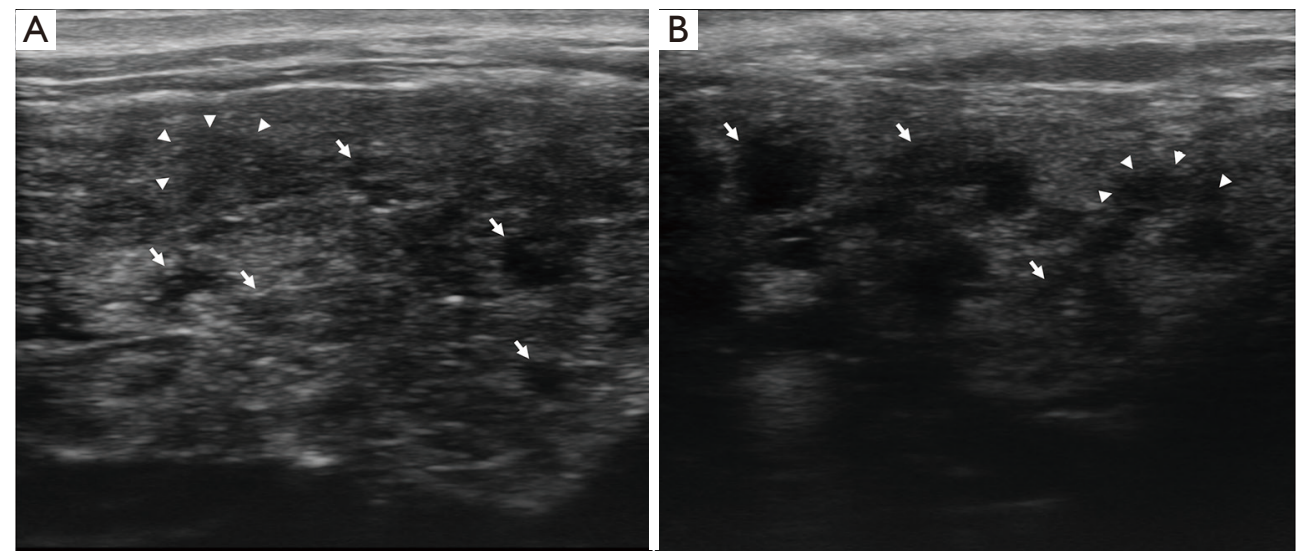

Figure 3 Severe alterations in the glandular parenchyma of parotid (A) and submandibular (B) glands in course of pSS depicted by using UHFUS with the $48 \mathrm{MHz}$ probe. Multiple single (arrows) or confluent (arrowheads) hypoechoic areas can be detected, along with disruption of normal glandular structure. pSS, primary Sjogren's syndrome; UHFUS, ultra-high frequency ultrasound.

\section{Minor SGs UFHUS}

Minor SGs are localized in the submucosal layer of the buccal mucosa, labial mucosa, lingual mucosa, soft/hard palate, and floor of the mouth (53). The study of minor SGs is of particular interest when considering labial localization. In fact, labial minor SGs are an important part of the diagnostic algorithm of pSS, as bioptic sampling is performed at this level to obtain histologic confirmation of diagnosis. In this sense, inferior labial mucosa represents an accessible site for surgical biopsy, due to a relatively superficial localization of the gland and a low risk of surgical complications. While minor SGs cannot be normally imaged with conventional US frequencies (53), UFHUS allows the evaluation of minor SGs thanks to the higher spatial resolution (up to $30 \mu \mathrm{m}$ ) provided by the $70 \mathrm{MHz}$ probe (19). Among all the minor SGs localizations, the inferior labial mucosa is the most accessible for UHFUS scanning. Given the superficial localization of the labial glands and the relatively small thickness of the labial mucosa, the US penetration of this probe-up to $10 \mathrm{~mm}-$ results adequate to investigate these structures.

We present our preliminary experience regarding UHFUS of the minor labial salivary glands (MLSGs), including our methodological approach, findings and 


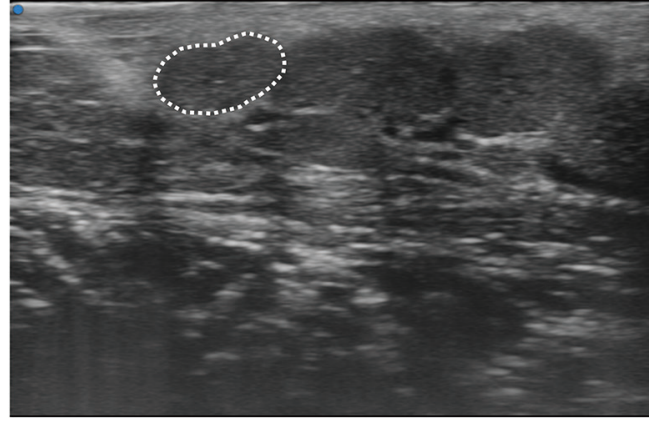

Figure 4 Normal labial minor salivary glands evaluated with UHFUS operating at $70 \mathrm{MHz}$. Similarly to major salivary glands, each minor labial salivary gland shows homogeneous parenchymal echostructure and defined and regular margins (dotted line). UHFUS, ultra-high frequency ultrasound.

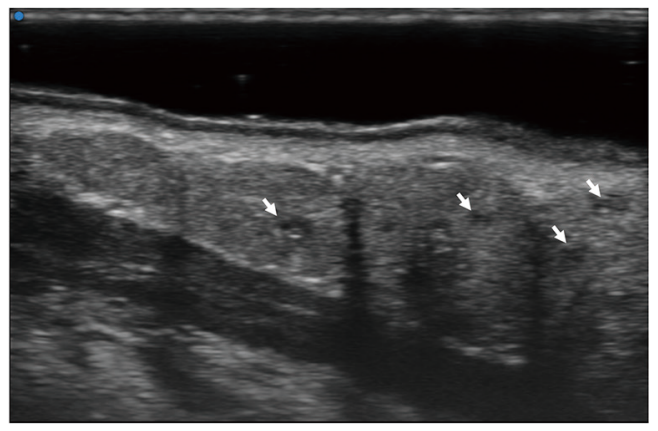

Figure 5 Mildly altered labial minor salivary glands depicted by using UHFUS with the $70 \mathrm{MHz}$ probe. In the initial stage of pSS, salivary glands usually preserve normal dimensions, although slight inhomogeneity and rare hypoechoic areas (arrows) can be detected. UHFUS, ultra-high frequency ultrasound; pSS, primary Sjogren's syndrome.

observations.

MLSGs UHFUS examination is carried out with the patient's mouth slightly open and the lower lip gently stretched out with a sterile gauze. First, a small amount of US gel is placed on the scanning surface of the transducer, which is then enveloped with a disposable probe-cover to avoid cross-infection during intraoral UHFUS scan. Subsequently, a sufficient amount of isotonic physiological solution is placed on the covered probe and on the labial mucosa to optimize the US transmission and to reduce reflection. Finally, the labial mucosa is scanned with longitudinal and transverse positioning of the probe respectively in the central, lateral left, and lateral right

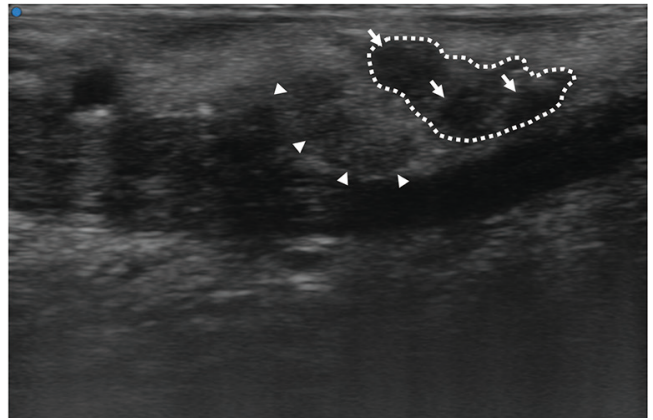

Figure 6 Severe alteration of labial minor salivary glands in course of pSS. Similarly to major salivary glands, minor salivary glands present irregular margins (dotted line) and extensive echostructural alterations, with hypoechoic areas (arrows), which in some cases appear confluent (arrowheads). Glands can be slightly decreased in terms of dimensions. pSS, primary Sjogren's syndrome.

compartment in order to provide a complete scan of the lip.

On UHFUS, MLSGs are depicted as roundish structures disposed in groups with characteristics of glandular parenchyma in terms of echostructure, as previously reported in the literature (19), appearing slightly hypoechoic compared to the hyperechoic submucosal connective tissue (Figure 4). Glandular dimensions and distribution vary through the different compartments, with numerous larger glands being localized in the lateral compartments and fewer smaller ones in the central area.

At UHFUS examination, normal MLSGs show a homogeneous echogenicity similar to major SGs. Despite the low amount of parenchyma in the MLSGs, alterations in the glandular echostructure can be detected, including parenchymal inhomogeneity, hypoechoic areas and hyperechoic foci/bands (Figures 5 and 6 ).

Based on a preliminary experience, Baldini et al. first demonstrated the feasibility of MLSGs UHFUS in 32 consecutive patients with clinically suspected pSS (54). All the patients were evaluated with a complete diagnostic work-up according to the ACR/EULAR criteria 2016, including LSGB (55). The pSS diagnosis was confirmed in $12 / 32(37.5 \%)$ cases. According to their results (54), no differences between patients with pSS and controls with noSS sicca syndrome were observed with UFHUS evaluation of MLSGs distribution and C/PDUS vascular parameters. On the contrary, a statistically significant increase of UHFUS inhomogeneity of glandular parenchyma $(\mathrm{P}=0.006)$ and a higher degree of fibrosis $(\mathrm{P}=0.01)$ was observed in the MLSGs of the lateral compartments (Figure 7) (54). This 


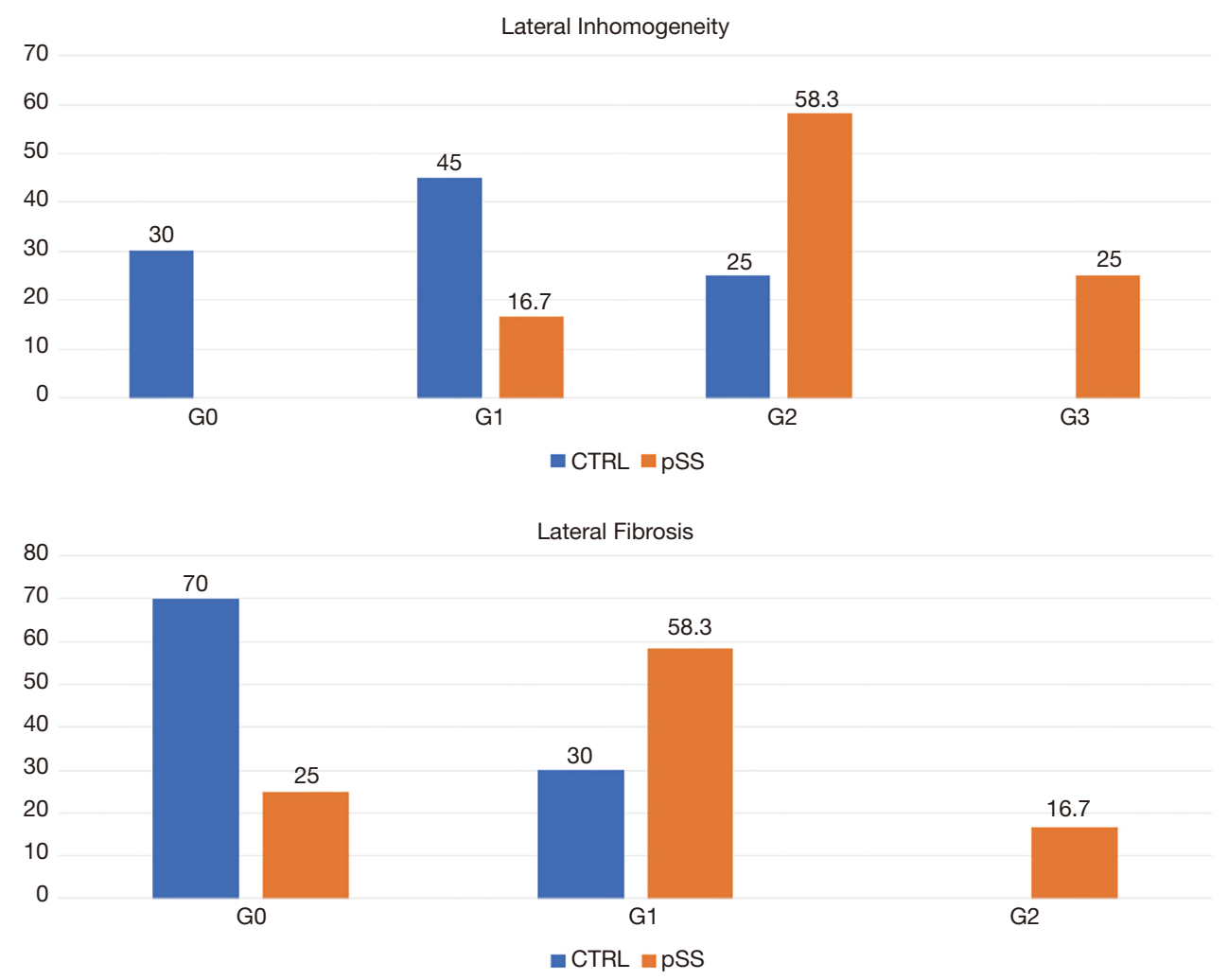

Figure 7 Bar graphs showing the statistically significant increase of UHFUS inhomogeneity of glandular parenchyma (P=0.006) and the higher degree of fibrosis $(\mathrm{P}=0.01)$ observed in the MLSGs of the lateral compartments. Image and data were retrieved from ref (54). CTRL, controls; pSS, patients with primary Sjogren syndrome; G0-G3 and G0-G2 refer to the degree of inhomogeneity and fibrosis, respectively. UHFUS, ultra-high frequency ultrasound; MLSG, minor labial salivary gland.

work paved the way for a novel application of UHFUS technique in the assessment of pSS, allowing for the first time a non-invasive and highly detailed evaluation of the MLSGs. Further studies are needed to confirm the potential role of MLSGs UHFUS in the pSS clinical setting.

The application of UHFUS technique for MLSGs evaluation presents some intrinsic limitations. Contrary to SG-UHFUS, a direct comparison between UHFUS and conventional US in MLSGs is not feasible, due to the limitation of the latter hindering minor SGs imaging. From a technical point of view, the US scan with the $70 \mathrm{MHz}$ probe is more difficult to perform due to the impact on the images of even minimal movements of the probe. The lack of dedicated probes may represent a limit especially in terms of invasiveness of the intraoral scan. The management of cross-infection risk is obviously an important issue which must be addressed by adopting adequate precautions in probe covering when performing intraoral UHFUS.
However, the disposable cover may cause artefacts in the superior part of the scan due to the presence of multiple interfaces for the US beam prior to reaching the target tissue.

For these reasons, a training period may be needed to achieve the manual skills required to correctly perform the examination.

\section{Future perspectives}

The application of UHFUS technique appears promising for several future perspectives in the SGs evaluation for pSS assessment, moving towards a more accurate integration of tissue and imaging biomarkers. On the one hand, the higher resolution of UHFUS in major SGs may have a role in the early diagnosis of pSS, thus improving management and follow-up of patients. On the other hand, the possibility of direct UHFUS evaluation of the MLSGs 

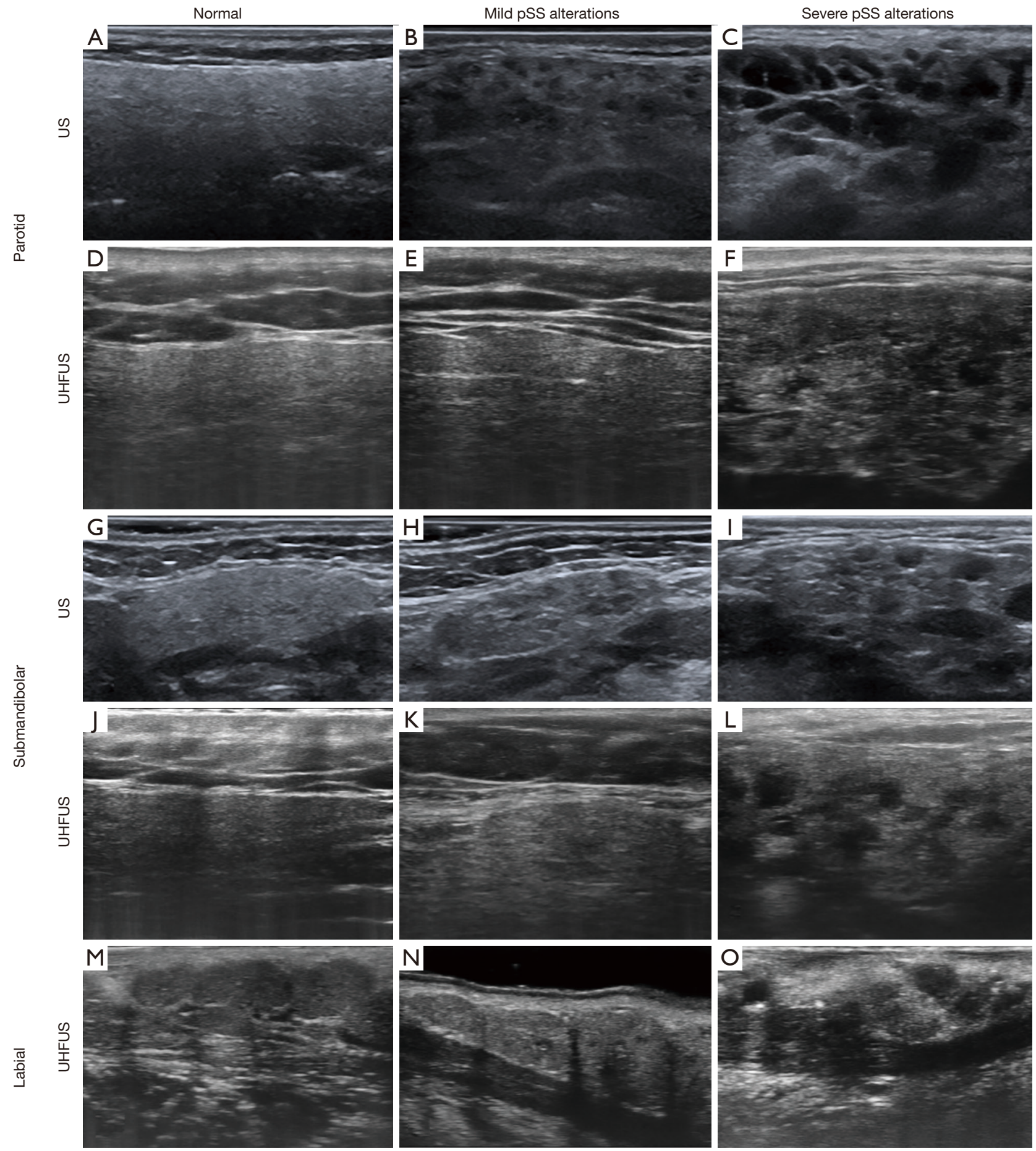

Figure 8 Overall comparison of the UHFUS visualisation of the normal appearance and the different degrees of alterations related to pSS in parotid (D,E,F), submandibular (J,K,L) and minor labial salivary glands (M,N,O). Comparative conventional US images for parotid (A,B,C) and submandibular $(\mathrm{G}, \mathrm{H}, \mathrm{I})$ glands are included. Images acquired in the university hospital of Pisa. pSS, primary Sjogren syndrome; US, ultrasound; UHFUS, ultra-high frequency ultrasound. 
may complete the diagnostic algorithm of pSS, in terms of giving additional information on the echostructure of these glands and of providing direct comparison with histology. The direct visualization of the MLSGs may improve presurgical planning for biopsies and subsequent histological examination, potentially reducing the number of inadequate biopsies. Moreover, the performance of comparative studies between UHFUS and histology may highlight a relevance for UHFUS investigation and potentially re-define the criteria for biopsy according to UHFUS-based parameters. If further studies will reveal the presence of a positive correlation between histology and UHFUS of minor SGs, pSS diagnostic algorithm will potentially be oriented to a less invasive approach limiting the biopsy of minor SGs to few selected cases.

\section{Conclusions}

This work aimed to present the current concepts of UHFUS as a novel technique for the evaluation of SGs by placing them in the state-of-the-art context of US applications in pSS, focusing on the pros and cons of this novel technique and its future potential applications.

Conventional US of major SGs appears a valid support for pSS diagnosis, although its role is still controversial. As for conventional US, UHFUS has the advantages of being safe, repeatable, and non-invasive. The high-resolution provided by the UHFUS thanks to the $48 \mathrm{MHz}$ and $70 \mathrm{MHz}$ probes allows to reach a high-detailed evaluation of the major and minor SGs. In Figure 8, the UHFUS aspect of normal major and minor SGs is shown, along with the different degrees of alterations related to pSS. For major SGs, comparative conventional US images are also provided (Figure 8).

UHFUS investigation of major SGs in the diagnostic algorithm of pSS seems promising, as the high resolution provided by the technique can be extremely valuable in detecting glandular alterations even at an early stage.

UHFUS of minor SGs represents a promising tool in the pSS work-up, as it is the only technique which allows a noninvasive evaluation of these structures with high resolution and high diagnostic confidence. Moreover, it could provide direct correlation between histology and echostructure, due to the bioptic sampling being performed on labial minor SGs.

This new technique applied to the major and minor SGs evaluation might lead to several potential improvements, including earlier diagnosis, reduction of unnecessary and inadequate biopsies and better management and followup of patients with pSS. However, given the small amount of preliminary data available, further studies are needed to confirm the role of UHFUS in the pSS clinical setting and research.

\section{Acknowledgments}

Funding: None.

\section{Footnote}

Provenance and Peer Review: This article was commissioned by the Guest Editor (Antonio Barile) for the series "Multimodality Advanced Imaging and Intervention in Gland Diseases" published in Gland Surgery. The article has undergone external peer review.

Reporting Checklist: The authors have completed the Narrative Review reporting checklist. Available at http:// dx.doi.org/10.21037/gs-20-529

Conflicts of Interest: All authors have completed the ICMJE uniform disclosure form (available at http://dx.doi. org/10.21037/gs-20-529). The series "Multimodality Advanced Imaging and Intervention in Gland Diseases" was commissioned by the editorial office without any funding or sponsorship. The authors have no other conflicts of interest to declare.

Ethical Statement: The authors are accountable for all aspects of the work in ensuring that questions related to the accuracy or integrity of any part of the work are appropriately investigated and resolved.

Open Access Statement: This is an Open Access article distributed in accordance with the Creative Commons Attribution-NonCommercial-NoDerivs 4.0 International License (CC BY-NC-ND 4.0), which permits the noncommercial replication and distribution of the article with the strict proviso that no changes or edits are made and the original work is properly cited (including links to both the formal publication through the relevant DOI and the license). See: https://creativecommons.org/licenses/by-nc-nd/4.0/.

\section{References}

1. Brito-Zerón P, Baldini C, Bootsma H, et al. Sjögren 
syndrome. Nat Rev Dis Prim 2016;2:16047.

2. Maciel G, Crowson CS, Matteson EL, et al. Prevalence of Primary Sjögren's Syndrome in a US Population-Based Cohort. Arthritis Care Res (Hoboken) 2017;69:1612-6.

3. Monsalve DM, Anaya JM. With minor salivary gland biopsy in Sjögren syndrome, is a negative result possible? J Rheumatol 2020;47:310-2.

4. Scotto di Santolo M, Massimo C, Tortora G, et al. Clinical value of high-resolution (5-17 MHz) echo-color Doppler (ECD) for identifying filling materials and assessment of damage or complications in aesthetic medicine/surgery. Radiol Med 2019;124:568-74.

5. Kim DW. Computed tomography features of the major salivary glands after radioactive iodine ablation in patients with papillary thyroid carcinoma. Radiol Med 2018;123:20-7.

6. Kim DW, Lee YJ, Ahn HS, et al. Comparison of ultrasonography and computed tomography for diagnosing diffuse thyroid disease: a multicenter study. Radiol Med 2018;123:515-23.

7. Izzetti R, Fantoni G, Gelli F, et al. Feasibility of intraoral ultrasonography in the diagnosis of oral soft tissue lesions: a preclinical assessment on an ex vivo specimen. Radiol Med 2018;123:135-42.

8. Caliskan E, Ozturk M, Bayramoglu Z, et al. Evaluation of parotid glands in healthy children and adolescents using shear wave elastography and superb microvascular imaging. Radiol Med 2018;123:710-8.

9. Agostini A, Borgheresi A, Mari A, et al. Dual-energy CT: theoretical principles and clinical applications. Radiol Med 2019;124:1281-95.

10. Kara M, Caliskan E, Atay G, et al. Shear wave elastography of parotid glands in pediatric patients with HIV infection. Radiol Med 2019;124:126-31.

11. Vogl TJ, Albrecht MH, Nour-Eldin NE din A, et al. Assessment of salivary gland tumors using MRI and CT: impact of experience on diagnostic accuracy. Radiol Med 2018;123:105-16.

12. Giurazza F, Corvino F, Cangiano G, et al. Sclerotherapy of peripheral low-flow vascular malformations: technical aspects and mid-term clinical outcome. Radiol Med 2018;123:474-80.

13. Hoffman HT, Pagedar NA. Ultrasound-Guided Salivary Gland Techniques and Interpretations. Atlas Oral Maxillofac Surg Clin North Am 2018;26:119-32.

14. Salaffi F, Carotti M, Iagnocco A, et al. Ultrasonography of salivary glands in primary Sjögren's syndrome: A comparison with contrast sialography and scintigraphy.
Rheumatology 2008;47:1244-9.

15. Vitali C, Carotti M, Salaffi F. Is it the time to adopt salivary gland ultrasonography as an alternative diagnostic tool for the classification of patients with Sjögren's syndrome? Comment on the Article by Cornec et al. Arthritis Rheum 2013;65:1950.

16. Cornec D, Jousse-Joulin S, Marhadour T, et al. Salivary gland ultrasonography improves the diagnostic performance of the 2012 American college of rheumatology classification criteria for sjögren's syndrome. Rheumatology (Oxford) 2014;53:1604-7.

17. Takagi Y, Sumi M, Nakamura H, et al. Ultrasonography as an additional item in the American college of Rheumatology classification of Sjögren's syndrome. Rheumatology (Oxford) 2014;53:1977-83.

18. Izzetti R, Vitali S, Aringhieri G, et al. The efficacy of Ultra-High Frequency Ultrasonography in the diagnosis of intraoral lesions. Oral Surg Oral Med Oral Pathol Oral Radiol 2020;129:401-10.

19. Izzetti R, Vitali S, Aringhieri G, et al. Discovering a new anatomy: exploration of oral mucosa with ultrahigh frequency ultrasound. Dentomaxillofac Radiol 2020;49:20190318.

20. Delli K, Dijkstra PU, Stel AJ, et al. Diagnostic properties of ultrasound of major salivary glands in Sjögren's syndrome: A meta-analysis. Oral Dis 2015;21:792-800.

21. Jousse-Joulin S, Milic V, Jonsson MV, et al. Is salivary gland ultrasonography a useful tool in Sjögren's syndrome? A systematic review. Rheumatology (Oxford) 2016;5 5:789-800.

22. Cornec D, Devauchelle-Pensec V, Saraux A, et al. Apport de l'échographie des glandes salivaires dans la prise en charge du syndrome de Sjögren : Où en sommes-nous ? Rev Med Interne 2016;37:186-94.

23. Zajkowski P, Ochal-Choi ska A. Standardy badania linianek - aktualizacja. J Ultrason 2016;16:175-90.

24. AIUM Practice Guideline for the Performance of Ultrasound Examinations of the Head and Neck. J Ultrasound Med 2014;33:366-82.

25. Carotti M, Salaffi F, Di Carlo M, et al. Diagnostic value of major salivary gland ultrasonography in primary Sjögren's syndrome: The role of grey-scale and colour/ power Doppler sonography. Gland Surg 2019;8:S159-67.

26. Luciano N, Ferro F, Bombardieri S, et al. Advances in salivary gland ultrasonography in primary Sjögren's syndrome. Clin Exp Rheumatol 2018;36:159-64.

27. Martire MV, Santiago ML, Cazenave T, et al. Latest Advances in Ultrasound Assessment of Salivary Glands in 
Sjögren Syndrome. J Clin Rheumatol 2018;24:218-23.

28. Jousse-Joulin S, Nowak E, Cornec D, et al. Salivary gland ultrasound abnormalities in primary Sjögren's syndrome: Consensual US-SG core items definition and reliability. RMD Open 2017;3:e000364.

29. Astorri E, Sutcliffe N, Richards PS, et al. Ultrasound of the salivary glands is a strong predictor of labial gland biopsy histopathology in patients with sicca symptoms. J Oral Pathol Med 2016;45:450-4.

30. El Miedany YM, Ahmed I, Mourad HG, et al. Quantitative ultrasonography and magnetic resonance imaging of the parotid gland: Can they replace the histopathologic studies in patients with Sjogren's syndrome? Joint Bone Spine 2004;71:29-38.

31. Salaffi F, Argalia G, Carotti M, et al. Salivary gland ultrasonography in the evaluation of primary Sjögren's syndrome. Comparison with minor salivary gland biopsy. J Rheumatol 2000;27:1229-36.

32. Theander E, Mandl T. Primary Sjögren's syndrome: Diagnostic and prognostic value of salivary gland ultrasonography using a simplified scoring system. Arthritis Care Res (Hoboken) 2014;66:1102-7.

33. Martinoli C, Derchi LE, Solbiati L, et al. Color Doppler sonography of salivary glands. AJR Am J Roentgenol 1994;163:933-41.

34. Chikui T, Yonetsu K, Izumi M, et al. Abnormal blood flow to the submandibular glands of patients with Sjögren's syndrome: Doppler waveform analysis. J Rheumatol 2000;27:1222-8.

35. Lee KA, Lee SH, Kim HR. Diagnostic and predictive evaluation using salivary gland ultrasonography in primary Sjögren's syndrome. Clin Exp Rheumatol 2018;36:165-72.

36. Carotti M, Salaffi F, Manganelli P, et al. Ultrasonography and colour Doppler sonography of salivary glands in primary Sjögren syndrome. Clin Rheumatol 2001;20:213-9.

37. Jousse-Joulin S, Gatineau F, Baldini C, et al. Weight of salivary gland ultrasonography compared to other items of the 2016 ACR/EULAR classification criteria for Primary Sjögren's syndrome. J Intern Med 2020;287:180-8.

38. Rong AJ, Fan KC, Golshani B, et al. Multimodal imaging features of intraocular foreign bodies. Semin Ophthalmol 2019;34:518-32.

39. Bard RL. High-Frequency Ultrasound Examination in the Diagnosis of Skin Cancer. Dermatol Clin 2017;35:505-11.

40. Oranges T, Vitali S, Benincasa B, et al. Advanced evaluation of hidradenitis suppurativa with ultra-high frequency ultrasound: A promising tool for the diagnosis and monitoring of disease progression. Skin Res Technol 2020;26:513-9.

41. Albano D, Aringhieri G, Messina C, et al. High-Frequency and Ultra-High Frequency Ultrasound: Musculoskeletal Imaging up to $70 \mathrm{MHz}$. Semin Musculoskelet Radiol 2020;24:125-34.

42. Aringhieri G, Vitali S, Rossi P, et al. The new frontier of imaging: The micron. Clin Exp Rheumatol 2018;36:169.

43. Dangardt F, Charakida M, Chiesa S, et al. Intimal and medial arterial changes defined by ultra-high-frequency ultrasound: Response to changing risk factors in children with chronic kidney disease. PLoS One 2018;13:e0198547.

44. Izzetti R, Vitali S, Oranges T, et al. Intraoral Ultra High Frequency Ultrasound study of oral lichen planus: A pictorial review. Skin Res Technol 2020;26:200-4.

45. Izzetti R, Vitali S, Gabriele M, et al. Feasibility of a combination of intraoral UHFUS and CBCT in the study of peri-implantitis. Oral Surg Oral Med Oral Pathol Oral Radiol 2019;127:e89-94.

46. Izzetti R, Vitali S, Aringhieri G, et al. Ultra-High Frequency Ultrasound, A Promising Diagnostic Technique: Review of the Literature and Single-Center Experience. Can Assoc Radiol J 2020;846537120940684.

47. Jasaitiene D, Valiukeviciene S, Linkeviciute G, et al. Principles of high-frequency ultrasonography for investigation of skin pathology. J Eur Acad Dermatol Venereol 2011;25:375-82.

48. Foster FS, Pavlin CJ, Harasiewicz KA, et al. Advances in ultrasound biomicroscopy. Ultrasound Med Biol 2000;26:1-27.

49. Filoux E, Mamou J, Aristizábal O, et al. Characterization of the spatial resolution of different high-frequency imaging systems using a novel anechoic-sphere phantom. IEEE Trans Ultrason Ferroelectr Freq Control 2011;58:994-1005.

50. Saied F, Włodkowska-Korytkowska M, Maślińska M, et al. The usefulness of ultrasound in the diagnostics of Sjögren's syndrome. J Ultrason 2013;13:202-11.

51. Wernicke D, Hess H, Gromnica-Ihle E, et al. Ultrasonography of salivary glands - A highly specific imaging procedure for diagnosis of Sjögren's syndrome. J Rheumatol 2008;35:285-93.

52. Baldini C, Luciano N, Tarantini G, et al. Salivary gland ultrasonography: A highly specific tool for the early diagnosis of primary Sjögren's syndrome. Arthritis Res Ther 2015;17:146.

53. Kessler AT, Bhatt AA. Review of the Major and Minor Salivary Glands, Part 1: Anatomy, Infectious, and 
Inflammatory Processes. J Clin Imaging Sci 2018;8:47.

54. Baldini C, Ferro F, Iodice V, et al. Ultra-High-Frequency Ultrasound of Labial Salivary Glands Highly Correlates With Histopathology in Primary Sjögren'S Syndrome. Ann Rheum Dis 2019;78:1162.

55. Shiboski CH, Shiboski SC, Seror R, et al. 2016 American

Cite this article as: Aringhieri G, Izzetti R, Vitali S, Ferro F, Gabriele M, Baldini C, Caramella D. Ultra-high frequency ultrasound (UHFUS) applications in Sjogren syndrome: narrative review and current concepts. Gland Surg 2020;9(6):22482259. doi: $10.21037 / g s-20-529$
College of Rheumatology/European League Against Rheumatism Classification Criteria for Primary Sjögren's Syndrome: A Consensus and Data-Driven Methodology Involving Three International Patient Cohorts. Arthritis Rheumatol 2017;69:35-45. 